\title{
An der Schnittstelle zwischen Ost und West Evangelische Kirchenmusik in Siebenbürgen
}

\author{
Ursula Philippi*
}

\author{
At the Point of Intersection between East and West: \\ Lutheran Church Music in Transylvania
}

Due to historical developments, Evangelical church music in Transylvania has changed significantly in recent decades. Major events that had an impact on this development are the establishment of the communist regime in Romania and the mass exodus of Germanspeaking Evangelical congregation members from most Transylvanian towns and villages. This poses a challenge for the Evangelical Church, who has to conserve and maintain largely unused and empty churches, as well as their assets, such as organs, sheet music, and other musical paraphernalia. However, the changed situation also has its benefits, since it requires an opening of the small Evangelical Church toward others. Music is particularly useful medium for this opening towards the non-Evangelical world, as it is not language-bound.

Keywords: Past, Present and Future of Evangelical church-music in Transylvania, Evangelical Church, organs, historical development, chances, adaptation.

Es sind fast 25 Jahre vergangen, seit die Wende von 1989 diesen Landstrich erfasst und dabei alle Bereiche des Lebens unglaublich verändert und durchgeschüttelt hat. Trotzdem geht von evangelischer Kirchenmusik hierzulande eine ungebrochene Faszination aus, um die Sie alle uns beneiden können! Im orthodoxen Umfeld, wo es unüblich ist, dass Gemeinden Choräle singen, dass Orgeln existieren und klingen, dass Chöre sich aufraffen, um große Werke der Musikliteratur einzustudieren, ist uns eine Aufmerksamkeit sicher, die einen großen Bonus darstellt. Lassen Sie mich Einiges zu den Chancen und Problemen erzählen, mit denen evangelische Kirchenmusik bei uns konfrontiert ist.

Die Vergangenheit, vielfach verklärt, ist hauptsächlich zu einem Gegenstand der Forschung geworden. Musikalien der evangelischen Kirche sind in Archiven verwahrt und werden studiert, herausgegeben, auf Tonträger eingespielt, manchmal auch in Sonderkonzerten live präsentiert. Einige Doktorarbeiten wurden schon verfass $\mathrm{t}^{1}$, und es warten noch zahlreiche weitere

* Prof. dr. Ursula Philippi, Professorin für künstlerisches Orgelspiel an der Staatlichen Musikhochschule Gheorghe Dima, Cluj (Klausenburg), Rumänien. Adresse: str. Morii 984 Ro 507165 Prejmer, judetul Brasov, Rumänien; e-mail: ursula@philippi.ro

1 Katalin Hanke, Zum Wirken der Komponisten Johann Sartorius, Vater und Sohn, Dissertation, 2007, Prof. Dr. Ferenc László, Staatliche Hochschule für Musik Gheorghe 
Themen auf Musikwissenschaftler. Wer sich damit beschäftigt, erkennt voller Respekt, wie fleißig die musikalischen Vorfahren waren. Auch wenn sie nicht an Bachs Kantaten heranreichten, belegen die siebenbürgischen Dicta, für jeden Sonntag und alle Feste von zahlreichen Kantoren im 18. und im 19. Jahrhundert komponiert, eine lebendige und gediegene musikalische Praxis. Auch wenn sie nicht so grandios wie in Norddeutschland waren, gehörten die siebenbürgischen Orgeln zum Inventar fast aller evangelischen Kirchen. Jede noch so kleine Gemeinschaft sorgte für ihre „Berufsmusiker“, die Adjuvanten, und war um die musikalische Ausbildung junger Menschen bemüht, die Orgel oder Violine spielen konnten. Choralbücher, freie Orgelstücke, musiktheoretische Abhandlungen wurden bei Kerzenschein abgeschrieben und als wertvolle Ausrüstungsgegenstände von Musiker zu Musiker weitergegeben. In Städten ließ man sich Orgelneubauten viel kosten: Vom Honorar des Instruments, das er 1671-1673 in der Hermannstädter evangelischen Stadtpfarrkirche erstellte, kaufte sich Johannes Vest aus Oberungarn ein Haus auf dem Großen Ring und erwarb das Bürgerrecht der Stadt. Die Gemeinde soll, so wird noch heute erzählt, einen Wald veräußert haben, um den kostspieligen Orgelbau mit seinem außerordentlichen Prospekt von zahlreichen musizierenden Gestalten zu finanzieren. Gymnasiasten hatten täglich zwei Stunden Musikunterricht. Das Gehalt des Hermannstädter Stadtkantors war zeitweilig das zweithöchste hinter dem Schulrektor. Die Beispiele kann man schier endlos fortsetzen.

Aber was bringt das für unsere Gegenwart? Sie ist geprägt vom anstrengenden Versuch, zu behalten, was möglich ist, zu bewahren, was uns heute so wertvoll und interessant erscheint. Dabei sind wir leider gezwungen, mit anzusehen, dass man nicht alles bewahren, schützen oder weiterführen kann. Viele Chöre, das Rückgrat der praktischen Kirchenmusik, gingen ein. Es verschwinden immer noch einige aus der Landschaft, so kürzlich der evangelische Chor der Gemeinde Bukarest. Man muss viel Fantasie und auch Energie einsetzen, um dem andauernden decrescendo irgendwie entgegenzuwirken.

Ganz besonders schwer fällt es mir, von den Orgeln zu erzählen. Wahrscheinlich wird höchstens jedes fünfte der über 200 Instrumente in evangelischen Kirchen noch gespielt und es sind allerhöchstens zehn Prozent der

Dima, Klausenburg/Cluj; Steffen Schlandt, Evangelisches Musikleben an der Scharzen Kirche zu Kronstadt, Dissertation, 2011, Prof. Dr. Ferenc László,Staatliche Hochschule für Musik Gheorghe Dima, Klausenburg/Cluj; Wolfgang Sand,Rudolf Lassel und das Musikleben Kronstadts an der Wende zum 20. Jahrhundert, Kludenbach, 1999; Ursula Philippi, Die Rolle der Orgel in der Liturgie der evangelischen Kirche Siebenbürgens, Dissertation, 2006, Prof. Dr. Ferenc László, Staatliche Hochschule für Musik Gheorghe Dima, Klausenburg/Cluj. 
Orgeln in einem guten Zustand. Wie soll man alle diese kleinen und größeren Instrumente retten? Wir besitzen eigentlich nur historische Orgeln, denn mit dem Ende des Zweiten Weltkriegs erlosch im kommunistischen Rumänien jede orgelbauerische Tätigkeit, bis auf Reparaturen und wenige Restaurierungen vorhandener Instrumente. Heute hat jede Stadtkirche schon mindestens eine Zweitorgel aus einem Dorf aufgenommen und restaurieren lassen. Staatliche Kultureinrichtungen übernehmen gerne wertvolle alte Instrumente, wenn man ihnen diese schenkt und auch instand setzt ${ }^{2} . .$. Der ganze große Rest ist Dieben, Nagetieren und dem Zahn der Zeit ausgesetzt. Leider geschieht das vor unseren Augen und Ohren! Glauben Sie es mir, jede Orgelreparatur, jedes Einweihungsfest nach einer Restaurierung ist bei uns ein großer Sieg. Ich durfte in diesem Jahr allein vier solcher wunderbarer Feste feiern! Vielleicht sind da doch mehr Chancen als geahnt? Das hat natürlich auch mit dem Geld zu tun. Nicht alle Gemeinden können oder wollen heute noch einen Wald verkaufen, damit sie teure Orgeln reparieren. Von Neubauten ist hier gar nicht die Rede! Bei diesen vier Orgelsanierungen des Jahres 2014 kam Geld von der politischen Gemeinde (Hermannstadt), von der eigenen kleinen Gemeinde und ihren Sponsoren (Broos), von den energischen Bemühungen einer Bankkauffrau (Kleinschenk) sowie von abenteuerlichen und nimmermüden Aktionen eines Pfarrers zur Gewinnung von Patenschaften und Sensibilisierung von Spendern bis hin zu einer Gruppe befreundeter Mohikaner in Wisconsin/USA (Hammersdorf).

Dabei bleibt die Frage, ob alle diese Orgeln außer bei der Einweihung noch gespielt werden? Längst fehlen Organisten und Organistinnen. Personalmangel ist heute unser wundester Punkt. Die deutsche Sprache, ein jahrhundertealtes evangelisches Spezifikum in Siebenbürgen, erweist sich in diesem Fall auch als Hindernis. Will ich bei der evangelischen Kirche als Orgelspieler einsteigen, ist es richtig schwer ohne die deutsche Sprache! Andererseits empört sich die Gemeinde, wenn der Chor zu Festtagen rumänische Lieder singt, weil Chorleiter und eventuell Chormitglieder diese als Muttersprache beherrschen. Nur in eingen Fällen geht das gut: Chorleiter oder Organistin engagieren sich für evangelische Kirchenmusik, auch wenn sie die Sprache in dieser Kirche nicht verstehen.

Und wie gestaltet sich die Zukunft? Im orthodoxen Umfeld hat evangelische Kirchenmusik durchaus ihre Chance. Orgelmusik ist beliebt und füllt

2 Die Staatliche Musikhochschule Klausenburg/Cluj erwarb 1992 eine historische Orgel von 1819 aus der evangelischen Kirche von Halwelagen/Hoghilag und nutzt diese als Ausbildungs- und Konzertinstrument. Die Restaurierung übernahm eine deutsche Firma aus Mitteln des Bundesministeriums des Innern der Bundesrepublik Deutschland. 
oft die Kirchen. Oratorien zu singen oder anzuhören ist eigentlich nur im evangelischen „Dunstkreis“ möglich, wenn man von den staatlich geförderten Radio- und Philharmoniechören absieht. Unser eigener Bachchor hier in Hermannstadt schwillt regelmäßig an, wenn ein oratorisches Werk geprobt wird. Ähnliches kann bestimmt auch der Kollege Steffen Schlandt aus Kronstadt berichten. Nur ein Bruchteil der Ausführenden gehört zur Gemeinde. Dem zahlreichen Publikum wird bei Konzerten eine Übersetzung angeboten. Diese muss oft selbst erstellt werden. Das ist bei betrachtenden Arien und Choraltexten des Barock eine echte Herausforderung! Aber man wird auch entschädigt:

BESPIEL NACHBARIN KONZERT (Es wird von einer Konzertgängerin berichtet, die dem Chorleiter begeistert erzählt, dass sie in der Aufführung der Johannespassion gewesen sei. Die Zeit sei nur so verflogen, und am Ende habe sie völlig überrascht festgestellt, dass bereits zwei Stunden vergangen seien.)

Eine weitere Chance hat evangelische Kirchenmusik, wenn es noch Künstler gibt, die sie gestalten und neu beleben. Da darf ich auf den Komponisten Hans Peter Türk ${ }^{3}$ hinweisen, der sich nicht zu schade ist, auch für Laienensembles zu schreiben. Nicht wenige seiner Werke sind für Orgeleinweihungen oder kirchliche Feste in evangelischen Kirchen komponiert. Türk hat auch einen Weg beschritten, der möglicherweise Chancen eröffnet: In einem der Orgelwerke (Toccata, komp. 1998) zitiert und verarbeitet er ein altes siebenbürgisches Volkslied (Ze Krúnen, ze Krünen vorm Borjerdor). Als Orgelspielerin ist es für mich mehr als interessant, dass sich solche Tendenzen verstärken, dass Elemente der Folklore, auch der rumänischen, Eingang finden in Orgelkompositionen. Es gibt schon einige Stücke ${ }^{4}$, und erstaunlich ist immer wieder der Erfolg solcher Werke bei Konzerten. Die Panflöte, ein rumänisches Volksmusikinstrument par excellence, ist ja einer der allernächsten Verwandten der Orgel. In unserem Kontext, wo die Orgel gleichgesetzt wird mit Exotik, mit fremder Konfession und anderer Sprache, ist dies ein möglicher Weg, sie in der Mehrheitskultur mit zu integrieren.

Gehört die Zukunft den Crossover-Projekten? Darauf wird selbstverständlich nur die Zukunft antworten. Wie viel Eigenes müssen wir aufgeben, um zu überleben? Und müssen wir uns davor fürchten? Ich kann hier nur

\footnotetext{
3 Hans Peter Türk, geb. 1940 in Kronstadt/Brasov, emeritierter Professor für Tonsatz an der Musikhochschule Klausenburg/Cluj, gehört zu den führenden Persönlichkeiten der rumänischen Komponistenszene.

4 Sigismund Toduța: Sieben Bearbeitungen rumänischer Colinde (Weihnachtlicher Volkslieder) für Orgel.
} 
anhand von Fallbeispielen die Situation erhellen. Großen Zulauf haben in einigen unserer Stadt-Gemeinden die Kinderchöre. Dabei sind verschwindend wenige Kinder evangelisch getauft. Alle aber kommen aus Schulen, in denen deutsch unterrichtet wird. Gelingt es, diese Kinder zu halten, zum Singen und Tanzen zu motivieren, dann können wunderbare Feste gefeiert werden: zu Erntedank, im Advent, zu jeder sich bietenden Gelegenheit. Die Erwachsenen im Gottesdienst erleben aktive und begeistert singende Kinder. Es tut jedes Mal weh, dann dennoch zu hören: Aber das sind Fremde, ihre Aussprache ist nicht die richtige, sie gehören doch nicht zu uns! Natürlich teile ich Ihre Ansicht: Davon soll man sich nicht entmutigen lassen. Die vielen Kinder, mit denen man Musicals und Weihnachtsspiele einstudieren kann, lohnen jede Anstrengung. Nur können Sie vielleicht auch verstehen, wenn Kolleginnen müde werden, und Kollegen resignieren, weil diese Abneigung gegen „die Anderen“ und „das nie Dagewesene“ nicht nachzulassen scheint.

Wie andere Kirchen auch, sehen evangelische Kirchen in Siebenbürgen ihre Chance unter anderem im Kulturbetrieb. Da kann es vorkommen, dass sich Kirchen füllen, die lange schon fast leer standen. Gemeinden denken um und widmen dieser Arbeit neuerdings ein Budget. Es ist oft die einzige Chance, die anderskonfessionellen Nachbarn in das eigene Gotteshaus zu bekommen. Kirchengebäude öffnen sich. Musik erklingt und wird von allen verstanden. Kritiker werfen uns vor: Kammer- oder Orchestermusik ist keine evangelische Kirchenmusik. Gemeinderäte beraten, wie weit sie die Musikprogramme kontrollieren und zensieren sollen. Darf es ohne das Vaterunser und einen Segen des Geistlichen ablaufen? Oder sollen wir an den finanziellen Aspekt denken, also Miete und Eintrittskarten im Auge haben? Davon könnte man eventuell die Orgel sanieren...

Sie werden jetzt lächeln und abwinken, weil Sie zu Recht sagen: Das sind alles Probleme, die wir vor Jahren und Jahrzehnten hinter uns gelassen haben. Die hier in Siebenbürgen sollen von uns lernen und nicht alles neu erfinden wollen. Wir sind Ihnen auch sehr dankbar, dass Sie Ihre Tagung in diesem Jahr, 25 Jahre nach der Begegnung in Rostock, hierher verlegt haben. Wir erhoffen uns und brauchen dringend Stärkung, Ermutigung und gute neue Ideen. Ich erinnere mich noch an ein Detail der Gespräche in Rostock im September 1989: Es entbrannte ein Streit über die Atmungen am Zeilenende bei evangelischen Chorälen, speziell bei denen des Genfer Psalters. Mir war damals gar nicht so bewusst, dass das ein wichtiges Problem ist. Ich fühlte mich sehr unwissend und konnte mich keiner Meinung anschließen. Daheim erzählte ich dann von den in Rostock gewälzten Problemen und niemand zeigte besonderes Interesse. Manche singen mit, manche ohne Atempause 
zwischen den Choralzeilen, bei uns wie wahrscheinlich auch bei Ihnen. Wir alle müssen heute, nach 25 Jahren, einem anderen Problem ins Auge schauen: Wer singt denn noch Choräle in der Kirche? Wir haben in der evangelischen Landeskirche Rumäniens seit etwa 10 Jahren ein zweisprachiges Gesangbuch. Bei vielen Gelegenheiten liegt das neue grüne Buch auf: Bitte, rumänisch sprechende Schwestern und Brüder, singt doch mit uns mit! Die Orgel führt den Gesang, die Menschen starren ins Gesangbuch und singen in der Regel nicht. Kommt wohl der Tag, dass man in unserer Kirche laut singen hören wird: „Laudă pe Domnul, o suflete și-a lui mărire“? (Lobe den Herren, den mächtigen König der Ehren)

Lassen Sie mich aber mit einem Beispiel schließen, das für mich, die ich auch junge Menschen aller Konfessionen im Orgelspiel unterrichte, einen Hoffnungsschimmer enthält: Anca, eine orthodoxe Studentin aus dem Banat, war für zwei Jahre im Master-Studiengang Orgel in meiner Klasse an der Staatlichen Musikhochschule Gheorghe Dima in Klausenburg/Cluj. Das orthodoxe Osterfest feiert sie immer in ihrem Heimatdorf Liebling mit der Familie. Um Mitternacht geht man zur Kirche. Ein Höhepunkt des Auferstehungsfestes ist die Licht-Zeremonie gefolgt vom Lied Christos a înviat din morți, einem der ganz wenigen Gesänge in der orthodoxen Kirche, wo alle Beteiligten laut mitsingen. Mit Zittern und Zagen wagte es Anca in einer Osternacht, sich an die Orgel zu setzen und dieses Lied mitzuspielen. Man nutzt nämlich in Liebling die aufgelassene evangelische Kirche für die orthodoxen Gottesdienste. Ja, Anca war so mutig und berauscht vom Ereignis, dass sie auch am Ende die Orgel ertönen ließ. Seitdem stimmt alljährlich zu Ostern in Liebling die Orgel mit ein in den Gesang der orthodoxen Gemeinde, niemand nahm Anstoß, und es ist die einzige Gelegenheit im Jahr, dass die Orgel der evangelischen Kirche von Liebling erklingt! 